The Psychological Record, 2008, 58, 585-596

\title{
THE PERCEIVED-THREAT BEHAVIORAL APPROACH TEST (PT-BAT): MEASURING AVOIDANCE IN HIGH-, MID-, AND LOW-SPIDER-FEARFUL PARTICIPANTS
}

\author{
Andy Cochrane, Dermot Barnes-Holmes, and Yvonne Barnes-Holmes \\ National University of Ireland Maynooth
}

\begin{abstract}
One hundred twenty female participants, with varying levels of spider fear were asked to complete an automated 8-step perceived-threat behavioral approach test (PT-BAT). The steps involved asking the participants if they were willing to put their hand into a number of opaque jars with an incrementally increasing risk of contact with a spider (none of the jars actually contained a spider). There was a negative correlation between the number of steps completed and self-reported spider fear as measured with the Fear of Spiders Questionnaire (FSQ). Additionally, the task discriminated between high, mid, and low fear on 2 behavioral measures: (a) the number of steps completed and (b) reported willingness to return and repeat the task. The automated procedure provides a psychological challenge with a high level of experimental control.
\end{abstract}

The behavioral approach test (BAT) is a commonly used behavioral assessment for specific phobias (Antony \& Swinson, 2000) and a popular objective measure of clinical progress following treatment such as exposure therapy (Garcia-Palacios, Hoffman, Carlin, Furness, \& Botella, 2002). BATs developed by individual research teams for particular studies tend to vary in terms of the number of steps and types of stimuli employed, and this variance can limit comparability among different sets of findings (Rose \& McGlynn, 1997). Furthermore, access to and management of the appropriate stimulus can be time-consuming or difficult to arrange (Meng, Kirkby, Martin, Gilroy, \& Daniels, 2004). There are some other potential difficulties associated with BATs in the context of both experimental and clinical work. For example, researchers or clinicians are usually required to be present during the BAT to give instructions and to record anxiety levels, but even if blind to the experimental condition, they may unwittingly influence the performance of the participants. Additionally, it could be argued that in vivo exposure to the phobic stimuli during the BAT may function as a part of the therapeutic intervention, making it difficult to disentangle the processes of change from pre- to posttreatment.

This research was supported by the Irish Research Council for the Humanities and Social Sciences through a Government of Ireland Scholarship to the first author.

Correspondence concerning this article should be addressed to Andy Cochrane, Department of Psychology, NUI Maynooth, Maynooth, Co. Kildare, Ireland. Phone: +353 (0) 7084765 E-mail: acochrane@nuim.ie 
A number of early studies explored the use of pictures of phobic stimuli, rather than in vivo exposure, as a rapid screening device for high versus low levels of fear. The general findings were that high-fearful participants took longer to decide to view each slide and looked at fewer slides overall relative to their low-fearful counterparts (e.g., spiders in Burchardt \& Lewis, 1977; snakes in Hayes, Nelson, \& Willis, 1982). More recently, Meng et al. (2004) tested two computer-presented BATs to determine if they could discriminate between high- and low-spider-fearful participants. Participants completed a slide BAT, a video BAT, and an 11-step live BAT (the order was counterbalanced across participants). The slide BAT required the participants to view pictures of a huntsman spider that increased in size over 11 steps. The video presented the 11 steps of a live BAT from the perspective of the participant. Both of the computer-presented BATs discriminated between low- and high-fear participants with respect to subjective anxiety scores, and the video BAT elicited higher maximum anxiety ratings from the fearful participants relative to both the slide and live BAT. Nevertheless, only the traditional live BAT discriminated between fear levels on the behavioral measure (i.e., the number of steps of the task completed), with the high-fear group completing significantly fewer steps relative to the low-fear group. Meng et al. did suggest that variables such as size, vividness, and movement of the images could be manipulated to improve the behavioral discrimination of the computer-presented BATs, but no further research has been conducted to test this suggestion.

The research discussed so far used visual images as stimuli, but a recent study suggested that it may not be necessary for participants to come into direct visual contact with their feared object to elicit a fear response (Castaneda \& Segerstrom, 2004). Spider-anxious participants, matched for baseline levels of fear and worry, were exposed to an imagined stimulus (a taped narrative), an opaque condition (being told that a box contained a spider), or a live tarantula in a glass cage. The opaque condition elicited the most physiological arousal, as measured by increases in heart rate and skin conductance, and this effect was particularly evident as the box was moved closer to the participants.

The BAT employed in the current study aimed to provide a relatively precise measure of approach/avoidance without the participant actually seeing or coming into physical contact with a spider. Furthermore, the fully automated procedure meant that the task could be undertaken without the researcher being present in the room, thus reducing any potential safety cues or social demands that may influence performance on the task. As in more traditional approach tests, a series of increasingly difficult tasks was constructed. The eight steps involved asking each participant if she was willing to put her hand into a number of opaque jars with a gradually increasing risk of contact with an Irish house spider. The participants were also told that the final jar contained a tarantula; this "extreme" element was included to avoid ceiling effects (Williams, 1985). Importantly, unbeknown to the participant, none of the jars contained a spider, and as such the challenge could be defined as involving a perceived threat because actual contact, either physical or visual, with a spider was not required. The current methodology is therefore named the PerceivedThreat Behavioral Approach Task (PT-BAT).

The primary purpose of the current study was to explore the use of the PT-BAT as a procedure that would elicit anxious responding among individuals who report differing levels of spider fear. Even though no "dangerous" spiders are native to the country in which the current study was conducted 
(Ireland), previous research has shown that spiders produce varying levels of fear and anxiety within the Irish population (Smyth, Barnes-Holmes, \& Forsyth, 2006). The question posed was, Would the PT-BAT discriminate among high-, mid- and low-spider-fearful participants as measured with the Fear of Spiders Questionnaire? (Szymanski \& O’Donohue, 1995).

\section{Method}

\section{Participants}

One hundred twenty Irish undergraduate students were randomly selected from a volunteer subject pool and were invited to participate. Spider fear is far more prevalent among females than males (Fredrikson, Annas, Fischer, \& Wik, 1996), and consequently, only female participants were recruited.

Those with a recent history of an anxiety disorder, panic attacks, phobic disorder, or use of any psychotropic medication were excluded from participation (1 participant being excluded on this basis). Furthermore, 3 individuals declined to participate on learning that the experiment involved potential contact with spiders. The mean age was 24.44 years (standard error $[\mathrm{SE}]=.706$ ), with a range of 17 to 52 years.

\section{Materials and Apparatus}

Fear of Spiders Questionnaire. The Fear of Spiders Questionnaire (FSQ; Szymanski \& O'Donohue, 1995) is an 18-item tool for assessing spider phobia. Participants rate their agreement with statements such as "If I came across a spider now I would leave the room" on a 7 -point Likert-type scale $(0=$ strongly disagree, 6 = strongly agree). The FSQ loads onto two factors: avoidance/help seeking and fear of harm. It has good psychometric properties (alpha coefficient = 0.92; Szymanski \& O'Donohue, 1995). Additionally, the FSQ is capable of assessing low levels of self-reported spider fear and thus is suitable for use with a nonphobic sample (Muris \& Merckelbach, 1996).

There does appear to be some inconsistencies in the use of either a 7-point (e.g., Muris \& Merckelbach, 1996) or 8-point scale (e.g., Huijding \& de Jong, 2007), making direct comparisons of fear levels across studies problematic. Nevertheless, using a 7-point format, Muris and Merckelbach report a mean score of $3(\mathrm{SD}=7.8)$ for nonphobics and 89.1 ( $\mathrm{SD}=19.6$ ) for spider-phobics.

Disgust Scale. The 32-item Disgust Scale (Haidt, McCauley, \& Rozin, 1994) is an index of sensitivity to disgust-eliciting stimuli. It includes two true-false and two disgust-rating items for each of the seven domains of disgust elicitors (food, animals, body products, sex, body envelope violations, death, and hygiene). Alpha coefficients for the eight subscales range from .34 to .64 (Haidt et al., 1994).

State anxiety measure. The Spielberger State-Trait Anxiety Inventory Form-Y (STAI-Y; Spielberger, 1983) is used to assess levels of anxiety in clinical and nonclinical populations. The current study employed the state questionnaire only, which consists of 20 statements; the respondent is asked to indicate how she or he feels at that moment $(1=$ not at all to $4=$ very much so). The manual reports good to excellent internal consistency for both scales (alpha coefficients between .86 and.95) for college samples.

Subjective measures. Three computer-presented sliding scales were used 
to rate (a) each step of the PT-BAT tasks as pleasant or unpleasant $(1=$ very pleasant; 100 = very unpleasant), (b) the intensity of the emotional intensity experienced when asked to complete each step ( $1=$ mild; $100=$ intense), and (c) unwillingness to undertake each step ( 1 = very willing; $100=$ very unwilling). The participants were asked to indicate their rating by moving a sliding scale pointer with the mouse. The sliding scales were presented after each step of the PT-BAT.

The PT-BAT. The task consisted of a series of eight jars laid side by side and enclosed in a wooden frame. Each jar was $26 \mathrm{~cm}$ tall and had an opening $10 \mathrm{~cm}$ in diameter. The opening of each jar was covered with yellow material to obscure any view of the contents. Two $6 \mathrm{~cm}$ slits, in a cross shape, were cut into the material, allowing a hand to pass into the jar. The lids of the jars were labeled as follows: Jar 1 Empty; Jar 2 Had spider inside, now empty; Jar 3 20\% chance of spider; Jar 4 40\% chance of spider; Jar 5 60\% chance of spider; Jar 6 80\% chance of spider; Jar 7 100\% chance of spider; Jar 8 Big spider.

The PT-BAT equipment was designed to monitor the presence or absence of a participant's hand in each of the jars. This monitoring was achieved as follows. A light source (18-candle, green light-emitting diode [LED]) was attached through the outside wall of the wooden frame, next to the first jar, and a light sensor (light-dependent resistor, 9,000-22,000 ohm range) was attached through the opposite wall of the frame, next to the last jar. A plastic magnifying lens inserted between the two middle jars focused the light source onto the sensor. The light and sensor were interfaced to the printer port of the computer, which was programmed in Visual Basic 6 to react when participants moved their hands in and out of the jars.

Additional stimuli. Four photographs of different spider species were presented on the computer screen at the beginning of the PT-BAT. These were downloaded from the Internet (available from the authors on request).

\section{Procedure}

The experiment was conducted in the experimental psychopathology laboratory in the Department of Psychology at National University of Ireland Maynooth. This room offered the advantage of an adjacent observation room, with a one-way mirror, where the experimenter remained during each stage of the experiment (i.e., the experimenter could observe the participant, but not vice versa). The experiment was presented on a Dell personal computer with a Pentium 4 processor and a 15-inch-diameter standard monitor.

The participants undertook the task individually and were provided with the same general information before signing an informed consent statement. They then completed the pre-experiment questionnaires. The experimenter checked that the participant was familiar with the use of the mouse and how to control the audio volume of the computer speakers. The experiment was videotaped, with the participant's consent, and the camcorder was started before the experimenter left the room.

PT-BAT. Participants were seated in front of the computer monitor with the PT-BAT equipment positioned on a table to their right. The first message presented on the computer screen instructed the participant to click on the button to see some pictures. Four pictures of spiders appeared individually on the screen for 2,000 ms each. The purpose of these photographs was to increase the salience of the subsequent PT-BAT for a nonclinical population. A message 
then appeared, prompting participants to follow the on-screen instructions and reminding them that they were free to stop at any stage.

When the participant clicked on the box labeled "Click here to Start," the following message appeared: "Please read all instructions carefully. The first container is empty-are you willing to put your open hand into the container up to your wrist for 30 seconds?" Two separate rectangular boxes, one in the lower right corner (labeled "Yes") and one in the lower left (labeled "No") were on-screen at the same time as the message. If the participant clicked on the "Yes" button, the screen cleared and then the following message appeared: "Please put your hand into the jar now. The 30 seconds will start when you hear the first message and you will be told when to take your hand out."

The light beam running through the jars was broken when the participant put her hand into the jar, and this interruption prompted the playing of an audio message: "The 30 seconds starts now." A computer-controlled timer automatically started, and on completion of the 30-second period a second message, "Take your hand out now," was played. If the participant took her hand out before the 30 seconds was up, the light circuit was restored and as a consequence the following audio message played automatically: "You've taken your hand out too early; please put it back in or click on the button to start the next task." (Participants who put their hand back into the jar on hearing this message $(n=5)$ were deemed to have successfully completed the step. If participants did not put their hand back into the jar $(n=2)$, the step was not counted.) The following message was also on-screen in a rectangular box with a red background: "ONLY click here when you are ready to continue with the NEXT task."

When the participant clicked on the continue-with-next-task button, the three sliding scales (pleasant/unpleasant; emotional intensity; unwillingness) appeared on the screen. Participants were instructed to rate their reactions to the previous jar task on the three sliding scales, using the mouse to slide the pointer. Once participants had completed these ratings, they clicked on a continue button at the center-bottom of the screen and the next jar task was presented. The same sequence was followed for the remaining seven jars but with a different screen message describing the content of each subsequent jar:

2. "The second jar is empty but has previously contained a house spider."

3. "There is a $20 \%$ chance that there is a house spider in the third container."

4. "There is a 40\% chance that there is a house spider in the fourth container."

5. "There is a 60\% chance that there is a house spider in the fifth container."

6. "There is an $80 \%$ chance that there is a house spider in the sixth container."

7. "There is a $100 \%$ chance that there is a house spider in the seventh container."

8. "There is a nonpoisonous tarantula in the eighth container."

If the participant clicked on the "No" button at any stage in the sequence, the three sliding-scale questions were presented. In effect, the participant 
completed the subjective ratings for each jar regardless of whether she put her hand in. Once the eight jar tasks and subjective ratings had been presented, the participant made a final rating on a sliding scale to indicate how willing she would be to come back and repeat the same experiment the following week ( 0 = very willing, 100 = very unwilling). A message then appeared on-screen, indicating that this part of the experiment was finished. The participants were fully debriefed and thanked for their cooperation.

\section{Results}

\section{Levels of Spider Fear}

The mean FSQ score was 23.48 ( $\mathrm{SE}=2.017$ ), with a range of $0-86$. On the basis of the range of FSQ scores in this study, the participants were divided into three groups for further analyses: a low-fear group (FSQ scores $<15$; $n=$ 50 ), a mid-fear group (FSQ scores $15-32 ; n=35$ ), and a high-fear group (FSQ scores $>32 ; n=35$ ). This grouping is similar to that used by Huijding and de Jong (2006), allowing for the fact that they used an 8-rather than 7-point FSQ scale.

\section{Pre-experiment Measures}

The mean scores (+SE) for the disgust scale and state anxiety are presented in Table 1. A series of one-way analyses of variance (ANOVAs) were conducted to determine whether the three fear groups differed on either of these measures. The ANOVA approached significance for disgust sensitivity, $F(2,104)=$ 2.894, $p=.0596$. Scheffé post hoc tests revealed that the high-fear group scored significantly higher on the disgust scale than the low-fear group $(p=.0187)$. The ANOVA for state anxiety revealed a significant effect, $F(2,114)=5.253, p=$ $.0066, \eta_{p}{ }^{2}=.084$. Post hoc analyses indicated that the high-fear group reported higher levels of state anxiety relative to the mid- $(p=.0066)$ and low-fear ( $p=$ $.0044)$ groups. The three groups did not differ significantly by age $(p>.2)$.

Table 1

Mean (+SE) for Pre-Experiment Questionnaires

\begin{tabular}{ccccccr}
\hline & \multicolumn{3}{c}{$\begin{array}{c}\text { High-fear } \\
\mathrm{n}=35\end{array}$} & \multicolumn{2}{c}{$\begin{array}{c}\text { Mid-fear } \\
\mathrm{n}=35\end{array}$} & \multicolumn{2}{c}{$\begin{array}{c}\text { Low-fear } \\
\mathrm{n}=50\end{array}$} \\
\hline FSQ & 53.35 & $(2.73)$ & 18.55 & $(.84)$ & 6.02 & $(.67)$ \\
Disgust sensitivity & 17.36 & $(.74)$ & 16.20 & $(.74)$ & 14.89 & $(.72)$ \\
STAI-Y & 41.72 & $(1.85)$ & 35.00 & $(1.60)$ & 35.21 & $(1.45)$ \\
Age (years) & 20.74 & $(1)$ & 22.79 & $(1)$ & 23.41 & $(1.40)$ \\
\hline
\end{tabular}

Note. FSQ = Fear of Spiders Questionnaire, SE = standard error, STAI-Y = Spielberger State-Trait Anxiety Inventory Form-Y.

\section{Number of PT-BAT Steps Completed}

The mean (+SE) number of steps completed by each group is presented in Table 2. The low-fear group completed the most steps and high-fear the least, with nearly $70 \%$ of the latter group completing less than 3 steps (Figure 1). A one-way ANOVA revealed a significant effect for number of jars completed, $F(2,117)=36.100, p<.0001, \eta_{p}^{2}=.38$. Scheffé post hoc comparisons indicated 
that the high-fear group completed significantly fewer steps than the mid- ( $p<$ $.0001)$ and low-fear $(p<.0001)$ groups. Additionally, the mid-fear group completed significantly fewer steps than the low-fear group $(p=.0076)$.

Table 2

Mean (+SE) Steps of PT-BAT Completed and the Subjective Ratings Given for the Last Completed Step

\begin{tabular}{crrrrrr}
\hline & \multicolumn{2}{c}{ High-fear } & \multicolumn{2}{c}{ Mid-fear } & \multicolumn{2}{c}{ Low-fear } \\
\hline Steps completed & 2.89 & $(.42)$ & 5.55 & $(.36)$ & 6.80 & $(.26)$ \\
Unpleasantness & 42.54 & $(5.29)$ & 45.72 & $(4.38)$ & 44.43 & $(3.74)$ \\
Emotional intensity & 41.25 & $(4.89)$ & 47.46 & $(4.24)$ & 46.51 & $(3.65)$ \\
Unwillingness & 44.15 & $(5.57)$ & 43.69 & $(4.48)$ & 43.11 & $(3.53)$ \\
Unwilling to return & 57.07 & $(6.02)$ & 49.15 & $(5.84)$ & 24.49 & $(3.96)$ \\
\hline
\end{tabular}

Note. Higher scores for subjective ratings and unwillingness to return indicate more unpleasant, more intense, and greater unwillingness. PT-BAT = Perceived-Threat

Behavioral Approach Test, SE = standard error.

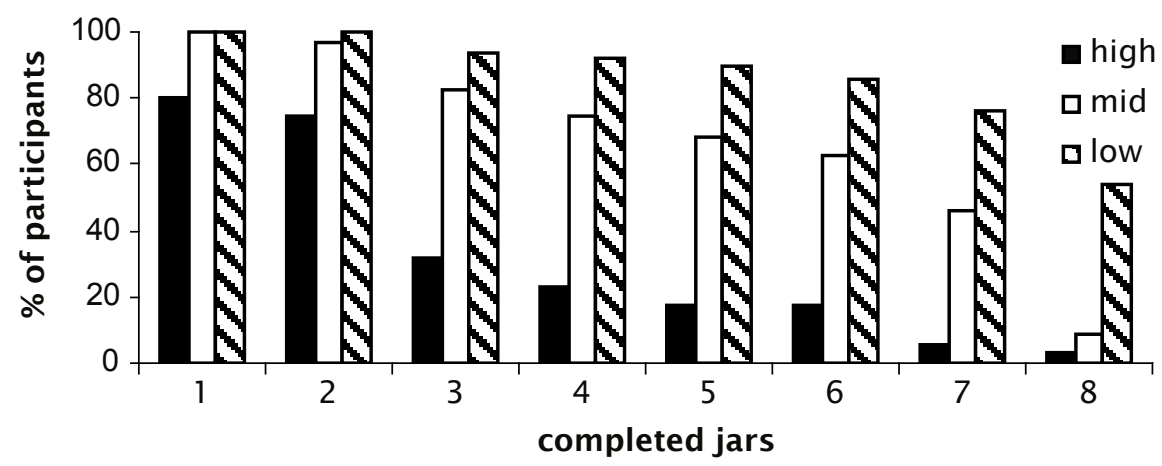

Figure 1. Percentage of each group completing the steps of the PT-BAT.

Thirty-one participants (25.83\% of all participants) completed all 8 steps of the PT-BAT and were predominantly from the low-fear group $(n=24)$. When these participants were removed from the analysis, the difference between the three groups remained significant, $F(2,85)=23.560, p<.0001$.

\section{Subjective Ratings During PT-BAT}

The three groups gave similar ratings in terms of unpleasantness, emotional intensity, and unwillingness for the last step they chose to complete (Table 3), which of course occurred at different stages in the jar sequence for each group. A series of one-way ANOVAs indicated that there were no significant differences among the groups for the subjective ratings reported after the last step completed (all $p s>.5$ ).

At the end of the PT-BAT, the participants were asked to indicate how willing they would be to return and complete the experiment at a later date (Table $3)$. A one-way ANOVA revealed a significant difference among the three groups, $\left.F(2,109)=12.052, p<.0001, \eta_{p}{ }^{2}=.181\right)$. Scheffé post hoc tests indicated that the low-fear group was significantly more willing to return than the high- ( $p<$ 
$.0001)$ and mid-fear ( $p=.0005$ ) groups; the difference between the high- and mid-fear groups was nonsignificant ( $p=.3132$ ).

Table 3

Pearson's Correlations for Questionnaires and PT-BAT Measures

\begin{tabular}{|c|c|c|c|c|c|c|c|c|}
\hline & FSQ & Disgust & STAIYY & $\begin{array}{l}\text { No. of Steps } \\
\text { Completed }\end{array}$ & $\begin{array}{l}\text { Unpleas- } \\
\text { antness }\end{array}$ & $\begin{array}{l}\text { Emotional } \\
\text { Intensity }\end{array}$ & $\begin{array}{l}\text { Unwill- } \\
\text { ingness }\end{array}$ & $\begin{array}{l}\text { Unwilling } \\
\text { to Return }\end{array}$ \\
\hline FSQ & & & & & & & & \\
\hline Disgust & .16 & & & & & & & \\
\hline STAI-Y & $.36^{* *}$ & -.07 & & & & & & \\
\hline $\begin{array}{l}\text { No. of steps } \\
\text { completed }\end{array}$ & $-.64 * *$ & $-.23 *$ & -.16 & & & & & \\
\hline $\begin{array}{l}\text { Unpleas- } \\
\text { antness }\end{array}$ & -.06 & .12 & -.04 & .06 & & & & \\
\hline $\begin{array}{l}\text { Emotional } \\
\text { Intensity }\end{array}$ & -.10 & .14 & -.03 & .12 & $.88^{* *}$ & & & \\
\hline $\begin{array}{l}\text { Unwilling- } \\
\text { ness }\end{array}$ & .04 & .11 & 10 & -.07 & $.62 * *$ & $.74 * *$ & & \\
\hline $\begin{array}{l}\text { Unwilling } \\
\text { to Return }\end{array}$ & $.36^{* *}$ & .02 & .11 & $-.38^{* *}$ & .18 & .15 & $.25 *$ & \\
\hline
\end{tabular}

Note. FSQ $=$ Fear of Spiders Questionnaire, PT-BAT $=$ Perceived-Threat Behavioral Approach Test, STAI-Y = Spielberger State-Trait Anxiety Inventory Form-Y.

$* * \mathrm{p}=.0001 .{ }^{*} \mathrm{p}<.01$.

\section{Questionnaire-PT-BAT Correlations}

The internal consistency of the FSQ using Cronbach's alpha was .961, a finding that is comparable with other studies using undergraduate samples (e.g., Muris \& Merckelbach, 1996). Pearson's correlations between the PT-BAT measures and relevant questionnaires are presented in Table 3. The results show that there were moderate to strong significant correlations between the FSQ and the two behavioral measures, namely the number of steps completed and willingness to return, supporting the convergent validity of the PT-BAT. The correlations among the pre-experiment screening questionnaires were between the FSQ and STAI-Y, and between disgust sensitivity and the number of steps completed. The three subjective ratings given during the PT-BAT correlated with each other, but only unwillingness to undertake each step correlated with one of the behavioral measures: unwillingness to return.

\section{Discussion}

The present study investigated the PT-BAT, an automated behavioral approach test during which participants do not come into physical or visual contact with a spider. The results indicate that the procedure is a useful measure of behavioral approach/avoidance in a nonclinical sample. Specifically, the PTBAT discriminated among low-, mid- and high-fear participants on two behavioral measures: (a) the number of steps completed and (b) reported willingness to return and repeat the task. Although there was some evidence of a ceiling effect, with about a quarter of the participants completing all eight steps of the PT-BAT, this was generally limited to the low-fear group, as measured with the FSQ.

Interestingly, the mean ratings given for the three subjective measures 
(unpleasantness, emotional intensity, unwillingness) for the last completed step of the PT-BAT were similar for the three groups. In other words, each group reported similar levels of discomfort at different points in the jar sequence. Thus, for example, the majority of the high-fear group reported levels of discomfort with jar 2 similar to those reported by the low-fear group with jar 8 . Clearly, the PT-BAT presented a psychological challenge to all three groups, and as would be predicted, the behavioral (avoidance) effect increased across the low-, mid-, and high-fear groups, respectively. On a related point, 4 participants in the high-fear group did not complete any steps of the PT-BAT; this subgroup had a mean FSQ of 66 (SE = 8.19), indicating a fear level approaching the phobic range (Muris \& Merckelbach, 1996). Thus the PT-BAT may pose a considerable challenge for persons diagnosed with spider phobia.

On balance, a possible strength of the PT-BAT is that the "contents" can be readily adjusted if too aversive for phobic participants or clients. For example, the perceived risk of contact could be increased more gradually (e.g., by $10 \%$ increments), and the number of steps to completion reduced or extended. Additionally, the type and size of the spider used can be readily changed to correspond with a prototypical spider feared by the participant. Furthermore, it is possible that the PT-BAT could be employed to assess avoidant behavior associated with other phobias of small animals or objects and, for example, to examine the relationship of such phobias with the fear of contamination or disgust sensitivity by manipulating the "contents" of the jars (e.g., including a "dead spider" jar).

The high-fear group in the current study reported higher levels of disgust sensitivity than did the low-fear group. There was also a weak correlation between the overall number of steps of the PT-BAT completed and the disgust scores. The relationship between phobias and disgust is currently unclear. Nevertheless, a core feature of spider phobia, it has been argued, may be the fear of coming into contact with a disgusting stimulus rather than the fear of being attacked and physically harmed (Davey, 1994). Indeed, one view suggests that disgust has a causal role in the development of phobias, in that certain animals elicit phobic responding because they are perceived as repulsive and potentially contaminating (e.g., see Davey, 1995). Furthermore, other authors suggest that disgust interacts with fear and may represent an increased vulnerability toward the development of phobias (e.g., Thorpe \& Salkovskis, 1998). In any case, disgust sensitivity may have contributed to avoidance during the PTBAT, by interacting with other factors that influence how individuals perform in unusual and potentially aversive situations (Woody \& Tolin, 2002).

The high-fear group also reported higher levels of state anxiety relative to the mid- and low-fear groups before starting the PT-BAT. The questionnaire was completed when the participants believed that the task involved potential contact with spiders, and thus this initial difference in anticipatory anxiety would appear to be specifically related to the task. Indeed, phobic responding is associated with a bias toward expecting aversive or traumatic outcomes after encounters with the phobic stimulus (Cavanagh \& Davey, 2000). Consistent with this expectancy bias model (Davey, 1995), participants in the high-fear group may have overestimated the risks involved in the PT-BAT and focused on the likelihood of an "aversive" outcome rather than the probability that a particular jar may not have contained a spider.

The high-fear group was the most unwilling to return and repeat the experiment, and their avoidant behavior may well contribute to the maintenance 
of any phobic-like symptoms (Antony \& Swinson, 2000). Similar issues regarding the retention of participants predisposed to avoidant behavior have been reported in other studies (e.g., Cochrane, Barnes-Holmes, Barnes-Holmes, Stewart, \& Luciano, 2006; Eifert \& Heffner, 2003), indicating that unwillingness to participate further could be included as a behavioral measure of avoidance.

The automated PT-BAT employed in the current study appears to provide a relatively robust measure of avoidance that discriminated between high, mid, and low levels of spider fear in a female nonclinical population. It is possible that a perceived threat represents a greater psychological challenge than an actual threat in the context of fear and phobia. A future study directly comparing performance on the PT-BAT with a traditional BAT may be useful in exploring this issue further.

Recent research conducted by our group has indicated that the PT-BAT can be used to assess behavioral and subjective changes following a therapeutic intervention for fear of spiders. Specifically, participants with varying levels of spider fear completed a baseline PT-BAT and were then exposed to a brief acceptance- or distraction-based intervention (using protocols adapted from McMullen et al., 2008). Both groups increased the number of steps completed on a postintervention PT-BAT but with a far larger effect size for the acceptance group. Furthermore, in a separate study, performance on the PT-BAT was shown to correlate with reaction-time-based responses on an implicit measure of spider fear, the Implicit Relational Assessment Procedure (Barnes-Holmes et al., 2006). Together these findings suggest that the PT-BAT may be employed effectively in future psychopathology research and shows potential for use with a clinical population.

\section{References}

ANTONY, M. M., \& SWINSON, R. P. (2000). Phobic disorders and panic in adults: a guide to assessment and treatment. Washington, DC: APA.

BARNES-HOLMES, D., BARNES-HOLMES, Y., POWER, P., HAYDEN, E., MILNE, R., \& STEWART, I. (2006). Do you really know what you believe? Developing the Implicit Relational Assessment Procedure (IRAP) as a direct measure of implicit beliefs. The Irish Psychologist, 32, 169-177.

BURCHARDT, C. J., \& LEVIS, D. J. (1977). The utility of presenting slides of a phobic stimulus in the context of a behavioral avoidance procedure. Behavior Therapy, 8, 340-346.

CASTANEDA, J. O., \& SEGERSTROM, S. C. (2004). Effect of stimulus type and worry on physiological response to fear. Journal of Anxiety Disorders, 18, 809-823.

CAVANAGH, K., \& DAVEY, G. C. L. (2000). UCS expectancy biases in spider phobics: underestimation of aversive consequences following fearirrelevant stimuli. Behaviour Research and Therapy, 38, 641-651.

COCHRANE, A., BARNES-HOLMES, D., BARNES-HOLMES, Y., STEWART, I., \& LUCIANO, C. (2007). Experiential avoidance and aversive visual images: response delays and event-related potentials on a simple matching task. Behaviour Research and Therapy, 45, 1379-1388.

DAVEY, G. (1994). The disgusting spider: the role of disease and illness in the perpetuation of fear of spiders. Society and Animals, 2, 17-25.

DAVEY, G. (1995). Preparedness and phobias: specific evolved associations or a generalised expectancy bias? Behavioral and Brain Sciences, 18, 289-325. 
EIFERT, G. H., \& HEFFNER, M. (2003). The effects of acceptance versus control contexts on avoidance of panic-related symptoms. Journal of Behavior Therapy and Experimental Psychiatry, 34, 293-312.

FREDRIKSON, M., ANNAS, P., FISCHER, H., \& WIK, G. (1996). Gender and age differences in the prevalence of specific fears and phobias. Behaviour Research and Therapy, 26, 241-244.

GARCIA-PALACIOS, A., HOFFMAN, H., CARLIN, A., FURNESS, T. A., \& BOTELLA, C. (2002). Virtual reality in the treatment of spider phobia: a controlled study. Behaviour Research and Therapy, 40, 983-993.

HAIDT, J., MCCAULEY, C., \& ROZIN, P. (1994). Individual differences in sensitivity to disgust: a scale sampling seven domains of disgust elicitors. Personality and Individual Differences, 16, 701-713.

HAYES, S. C., NELSON, R. O., \& WILLIS, S. E. (1982). Visual avoidance: the use of slides in a convenient measure of phobic avoidance. Behavioral Assessment, 4, 211-217.

HUIJDING, J., \& DE JONG, P. J. (2006). Specific predictive power of automatic spider-related affective associations for controllable and uncontrollable fear responses toward spiders. Behaviour Research and Therapy, 44, 161-176.

MCMULLEN, J., BARNES-HOLMES, D., BARNES-HOLMES, Y., STEWART, I., LUCIANO, C., \& COCHRANE, A. (2008). Acceptance versus distraction: brief instructions, metaphors, and exercises in increasing tolerance for self-delivered electric shocks. Behaviour Research and Therapy 46, 122-129.

MENG, C. T. T., KIRKBY, K. C., MARTIN, F., GILROY, L. J., \& DANIELS, B. A. (2004). Computer-delivered behavioural avoidance tests for spider phobia. Behaviour Change, 21, 173-185.

MURIS, P., \& MERCKELBACH, H. (1996). A comparison of two spider fear questionnaires. Journal of Behavioural Therapy and Experimental Psychiatry, 27, 241-244.

ROSE, M. P., \& MCGLYNN, F. D. (1997). Towards a standard experiment for studying post-treatment return of fear. Journal of Anxiety Disorders, 11, 263-277.

SMYTH, S., BARNES-HOLMES, D., \& FORSYTH, J. P. (2006). A derived transfer of simple discrimination functions in spider fearful and non-fearful participants. Journal of the Experimental Analysis of Behavior, 85, 223-246.

SPIELBERGER, C. D. (1983). State-Trait Anxiety Inventory (Form Y). Redwood City, CA: Mind Garden.

SZYMANSKI, J., \& O’DONOHUE, W. (1995). Fear of spiders questionnaire. Journal of Behavior Therapy and Experimental Psychiatry, 26, 31-34.

THORPE, S. J., \& SALKOVSKIS, P. M. (1998). Studies on the role of disgust in the acquisition and maintenance of specific phobias. Behaviour Research and Therapy, 36, 877-893.

WILLIAMS, S. L. (1985). On the nature and measurement of agoraphobia. Progress in Behavior Modification, 19, 109-144.

WOODY, S. R., \& TOLIN, D. F. (2002). The relationship between disgust sensitivity and avoidant behavior: studies of clinical and nonclinical samples. Journal of Anxiety Disorders, 16, 543-559. 
